\title{
State Measurements with Short Laser Pulses and Lower-Efficiency Photon Detectors
}

\author{
Almut Beige and Gerhard C. Hegerfeldt] \\ Institut für Theoretische Physik \\ Universität Göttingen \\ Bunsenstr. 9 \\ D-37073 Göttingen, Germany
}

\begin{abstract}
It has been proposed by Cook (Phys. Scr. T 21, 49 (1988)) to use a short probe laser pulse for state measurements of two-level systems. In previous work we have investigated to what extent this proposal fulfills the projection postulate if ideal photon detectors are considered. For detectors with overall efficiency less than 1 complications arise for single systems, and for this case we present a simple criterion for a laser pulse to act as a state measurement and to cause an almost complete state reduction.
\end{abstract}

\section{Introduction}

The outcome of measurements is often described in an idealized way by the projection postulate with its state vector reduction. The current form of the projection postulate is due to Lüders [1] and for degenerate eigenvalues it differs from that of von Neumann [2]. For non-degenerate eigenvalues it is implicitly contained in Dirac's book [3]. For the simple case of a two-level system in a state $|\psi\rangle$ and for a measurement of the energy the projection postulate states that, with probability $|\langle 1 \mid \psi\rangle|^{2}$, one finds $E_{1}$ and the state is changed to $|1\rangle$; correspondingly for $E_{2}$. In this simple case a measurement of the energy can also be regarded as a measurement of the eigenstates $|1\rangle$ and $|2\rangle$.

Cook [4] proposed to perform a measurement of the states $|1\rangle$ and $|2\rangle$ of such a two-level system by means of a short laser pulse which drives the transition between level 1 and an additional, rapidly decaying, level 3 (see Fig. 1). For a single system, initially in a superposition of $|1\rangle$ and $|2\rangle$, the short laser pulse may, or may not, lead to the emission of fluorescence photons. In the former case the system was taken to be in $|1\rangle$ after the end of the pulse (and after a short transient decay time for level 3), since right after each photon emission it is in the ground state. In the latter case the system was taken to be in $|2\rangle$. This was used in an experiment on the quantum Zeno effect [5].

In two previous papers [6, 7] we have investigated in how far this proposal realizes a measurement to which the projection postulate can be applied. Related work appeared in Refs. [8, 9, 10, 11, 12, 13]. It was shown in Ref. [6] that, under the condition of no photon emission during a pulse, the reduced (or conditional) time development [14, 15, 16, 17, 18] carries the state of the system ever closer to $|2\rangle$. The quality of this reduction to $|2\rangle$ can be expressed by a single combination of the pulse parameters, namely $N_{\mathrm{e}}\left(\tau_{\mathrm{p}} ;|1\rangle\right)$, the average number of photons emitted during a pulse of length $\tau_{\mathrm{p}}$ when one starts from $|1\rangle$ as initial state. $N_{\mathrm{e}}$ can be calculated from the fluorescence rate of an ensemble of such systems. It was shown in Ref. [6] that for $N_{\mathrm{e}}$ as low as 5 or 10 one has an excellent reduction to $|2\rangle$.

*e-mail: beige@theorie.physik.uni-goettingen.de

${ }^{\dagger}$ e-mail: hegerf@theorie.physik.uni-goettingen.de 
In quantum mechanics it is in principle not legitimate to speak about emission of a photon unless it is observed (detected). However, it was pointed out in Ref. [14] and Ref. [19] that in questions of photon statistics it makes no difference theoretically whether or not the photons are monitored over all space at rapidly repeated time intervals. Emitted photons are then those which would be detected by ideal detectors in the whole space.

The discussion in Refs. [6, 7] is based on such ideal detectors. With non-ideal detectors problems arise in the reduction of the state of a single system when no fluorescence photons are detected during a pulse, because this null detection may be due either to no emission at all or to a failure of the detector to record emitted photons. In the former case the system is in $|2\rangle$ at the end of the pulse, while in the latter case it is in $|1\rangle$. Hence the system has to be described by a density matrix with a $|2\rangle\langle 2|$ and a $|1\rangle\langle 1|$ contribution. For a good reduction to $|2\rangle$ the latter, the "non-reduced part" should be small, and the aim of this paper is to give a simple criterion to ensure this.

A real detector may miss a photon because of low quantum efficiency or because it operates only in a smaller solid angle円. We define the overall efficiency $\eta^{\prime}$ as the ratio of detected vs. emitted photons.

For a system initially in the ground state, we denote by $N_{\mathrm{d}}\left(\tau_{\mathrm{p}} ;|1\rangle\right) \equiv \eta^{\prime} N_{\mathrm{e}}\left(\tau_{\mathrm{p}} ;|1\rangle\right)$ the average number of fluorescence photons detected during a pulse. Experimentally this is easy to determine from the observed fluorescence of an ensemble ("gas") of systems, and one can adjust the pulse parameters to realize any value of $N_{\mathrm{d}}$ for a given detector. In a simplified derivation we will show in Section 3 of this paper that for initial state $|\psi\rangle$ the non-reduced part is approximately

$$
|\langle 1 \mid \psi\rangle|^{2} \mathrm{e}^{-N_{\mathrm{d}}}
$$

for small overall efficiency $\eta^{\prime}$. However, this simple derivation gives no insight on how good the approximation is and how small $\eta^{\prime}$ has to be. Therefore we show in the precise treatment of Section 4 that the non-reduced part is smaller than

$$
|\langle 1 \mid \psi\rangle|^{2}\left(1+\frac{2}{3} \eta^{\prime}\right) \mathrm{e}^{-N_{\mathrm{d}}}
$$

for $\eta^{\prime} \leq 1 / 3$ and $N_{\mathrm{d}} \geq 1$, provided $N_{\mathrm{e}}$ is sufficiently large, e.g. 5 , to ensure reduction in the case of ideal detectors. This is a strict bound from above; a strict bound from below is given at the end of Section 4 in Eq. (57). If the initial state is a density matrix $\rho$ instead of a pure state $|\psi\rangle$, then $|\langle 1 \mid \psi\rangle|^{2}$ is replaced by $\rho_{11}$. We derive Eq. (2) by Laplace transforms. Similar techniques have been used in Ref. [22].

\section{Basics and previous results}

We consider a $\mathrm{V}$ system as in Fig. 1, with level 2 (meta-)stable and level 3 rapidly decaying (Einstein coefficient $A$ ). The laser pulse has Rabi frequency $\Omega$ and is tuned to the $1-3$ transition. In the quantum jump approach (or Monte-Carlo wave function approach, or quantum trajectories) [14, 15, 16, 17, 18, 23] it is shown that under the condition of no photon emission the time development of a system is given by a reduced (or conditional) Hamiltonian. In our case it is given in an interaction picture by [6]

$$
H_{\text {red }} \equiv-\mathrm{i} \hbar M=\frac{\hbar}{2}\{\Omega(|1\rangle\langle 3|+| 3\rangle\langle 1|)-\mathrm{i} A|3\rangle\langle 3|\}
$$

\footnotetext{
${ }^{1}$ Solid angles smaller than $4 \pi$ have been considered in Ref. [20, 21], where in one solid angle photons were counted, while in the other solid angle the light was spectrally analyzed.
} 
The eigenvalue of $M$ are $\lambda_{2}=0$ (with eigenvector $|2\rangle$ ) and

$$
\lambda_{1,3}=\frac{1}{4}\left(A \mp \sqrt{A-4 \Omega^{2}}\right) .
$$

For initial state $|\psi\rangle$,

$$
|\psi\rangle=\alpha_{1}|1\rangle+\alpha_{2}|2\rangle
$$

the conditional time development is then

$$
\mathrm{e}^{-i H_{\mathrm{red}} \tau / \hbar}|\psi\rangle=\alpha_{2}|2\rangle+\alpha_{1}\left\{\frac{\lambda_{1} \mathrm{e}^{-\lambda_{3} \tau}-\lambda_{3} \mathrm{e}^{-\lambda_{1} \tau}}{\lambda_{1}-\lambda_{3}}|1\rangle+\frac{\mathrm{i}}{2} \Omega \frac{\mathrm{e}^{-\lambda_{1} \tau}-\mathrm{e}^{-\lambda_{3} \tau}}{\lambda_{1}-\lambda_{3}}|3\rangle\right\} .
$$

The probability for no photon emission until time $\tau$, denoted by $P_{0}^{\mathrm{e}}(\tau ;|\psi\rangle)$, is given by

$$
P_{0}^{\mathrm{e}}(\tau ;|\psi\rangle)=\| \mathrm{e}^{-\mathrm{i} H_{\mathrm{red}} \tau / \hbar}|\psi\rangle \|^{2}
$$

From Eq. (đ) one obtains

$$
P_{0}^{\mathrm{e}}(\tau ;|\psi\rangle)=|\langle 2 \mid \psi\rangle|^{2}+|\langle 1 \mid \psi\rangle|^{2} P_{0}^{\mathrm{e}}(\tau ;|1\rangle) .
$$

The probability density for the first photon emission is

$$
\begin{aligned}
w_{1}^{\mathrm{e}}(\tau ;|\psi\rangle) & =-\frac{\mathrm{d}}{\mathrm{d} \tau} P_{0}^{\mathrm{e}}(\tau ;|\psi\rangle) \\
& =|\langle 1 \mid \psi\rangle|^{2} w_{1}^{\mathrm{e}}(\tau ;|1\rangle)
\end{aligned}
$$

since the $|\langle 2 \mid \psi\rangle|^{2}$ term in Eq. (8) drops out. In particular, for an atom with initial state $|1\rangle$ one has

$$
P_{0}^{\mathrm{e}}(\tau ;|1\rangle)=\frac{1}{4} \Omega^{2}\left(\frac{\mathrm{e}^{-\lambda_{1} \tau}-\mathrm{e}^{-\lambda_{3} \tau}}{\lambda_{1}-\lambda_{3}}\right)^{2}+\left(\frac{\lambda_{1} \mathrm{e}^{-\lambda_{3} \tau}-\lambda_{3} \mathrm{e}^{-\lambda_{1} \tau}}{\lambda_{1}-\lambda_{3}}\right)^{2}
$$

and

$$
w_{1}^{\mathrm{e}}(\tau ;|1\rangle)=\frac{1}{4} \Omega^{2} A\left(\frac{\mathrm{e}^{-\lambda_{1} \tau}-\mathrm{e}^{-\lambda_{3} \tau}}{\lambda_{1}-\lambda_{3}}\right)^{2} .
$$

If $\tau_{\mathrm{p}}$, the duration of the pulse, satisfies

$$
\tau_{\mathrm{p}} \gg \max \left\{1 / A ; A / \Omega^{2}\right\}
$$

then for $\tau=\tau_{\mathrm{p}}$ the exponentials in Eq. (6) can be neglected and the state is proportional to $|2\rangle$, with probability

$$
P_{0}^{\mathrm{e}}\left(\tau_{\mathrm{p}} ;|\psi\rangle\right)=|\langle 2 \mid \psi\rangle|^{2}
$$

just as with the projection postulate. In this way one can understand why a system without emissions during a pulse ends up in the state $|2\rangle$.

With the complementary probability $1-|\langle 2 \mid \psi\rangle|^{2}=|\langle 1 \mid \psi\rangle|^{2}$ an atom will emit photons during a pulse and will be in a superposition of $|1\rangle$ and $|3\rangle$ at its end, at time $\tau_{\mathrm{p}}$. The $|3\rangle$ component will then either lead to the emission of an additional photon or the reduced time development (now with $\Omega=0$ ) leads to its vanishing (without emission). In both cases the state will be in $|1\rangle$, after a transient time $\tau_{\text {tr }}$ whose duration is several multiples of $A^{-1}$.

Analogously, for a system with no emission until $\tau_{\mathrm{p}}+\tau_{\text {tr }}$ the tiny $|3\rangle$ component, still present at time $\tau_{\mathrm{p}}$, has vanished by then and the remaining tiny $|1\rangle$ component of $\exp \left\{-\mathrm{i} H_{\text {red }} \tau_{\mathrm{p}} / \hbar\right\}|\psi\rangle$ in Eq. (6) is the part which is not reduced to $|2\rangle$. This can be estimated more precisely in terms of $N_{\mathrm{e}}\left(\tau_{\mathrm{p}} ;|1\rangle\right)$. The latter is obtained by integrating the photon rate $A \rho_{33}(\tau)$ where $\rho_{33}(\tau)$ is 
given by the Bloch equations for the driven 1-3 two-level system with $\rho_{11}(0)=1$ [24]. This gives

$$
N_{\mathrm{e}}(\tau ;|1\rangle)=\frac{\Omega^{2}}{A^{2}+2 \Omega^{2}}\left(A \tau-\frac{3 A^{2}}{A^{2}+2 \Omega^{2}}+\frac{2 A}{A^{2}+2 \Omega^{2}} \cdot \frac{\mu_{1}^{2} \mathrm{e}^{-\mu_{2} \tau}-\mu_{2}^{2} \mathrm{e}^{-\mu_{1} \tau}}{\mu_{1}-\mu_{2}}\right)
$$

where

$$
\mu_{1 / 2}=\frac{1}{4}\left(3 A \mp \sqrt{A^{2}-16 \Omega^{2}}\right) .
$$

For $N_{\mathrm{e}} \geq 1$ the contribution of the exponential terms is negligible. (For weak driving the time for the first emission is long which makes $\exp \left(-\mu_{i} \tau\right)$ small; for strong driving the factor in front of the last term is small.) We can and will therefore use, for $N_{\mathrm{e}} \geq 1$,

$$
N_{\mathrm{e}}\left(\tau_{\mathrm{p}} ;|1\rangle\right)=\frac{\Omega^{2}}{A^{2}+2 \Omega^{2}}\left(A \tau_{\mathrm{p}}-\frac{3 A^{2}}{A^{2}+2 \Omega^{2}}\right) .
$$

With this one can express $\tau_{\mathrm{p}}$ by $N_{\mathrm{e}}\left(\tau_{\mathrm{p}} ;|1\rangle\right)$ (and the remaining parameters) and can insert it for $\tau$ on the r.h.s. of Eq. (6). A graphical evaluation then yields as upper bound for the non-reduced part

$$
\left|\left\langle 1\left|\exp \left\{-\mathrm{i} H_{\mathrm{red}} \tau_{\mathrm{p}} / \hbar\right\}\right| \psi\right\rangle\right|^{2} \leq|\langle 1 \mid \psi\rangle|^{2} \mathrm{e}^{-N_{\mathrm{e}}\left(\tau_{\mathrm{p}} ;|1\rangle\right)} .
$$

Thus in the case of no photon emission during a pulse the reduction of $|\psi\rangle$ to $|2\rangle$ is already excellent if the parameters of the pulse are such that $N_{\mathrm{e}}\left(\tau_{\mathrm{p}} ;|1\rangle\right) \geq 5$, i.e. if the average number of photons emitted during the pulse by an atom initially in the ground state is 5 or more. The estimate in Eq. (17) can be improved substantially for strong driving [6].

\section{Simplified derivation}

We now incorporate a non-ideal detector of overall efficiency $\eta^{\prime}<1$. With initial state $|\psi\rangle$ as in Eq. (5) we denote by $P_{0}^{\mathrm{d}}(\tau ;|\psi\rangle)$ the probability of no detection of fluorescence photons until time $\tau$ for a single system. With probability $P_{0}^{\mathrm{e}}\left(\tau_{\mathrm{p}} ;|\psi\rangle\right)$ no photon is emitted during a pulse, and we assume in the following that for ideal detectors the non-reduced part is negligible, i.e. the corresponding state is $|2\rangle$. With probability $P_{0}^{\mathrm{d}}-P_{0}^{\mathrm{e}}$ photons are emitted, but missed by the non-ideal detector, and in this case the state is $|1\rangle$, after a transient decay time $\tau_{\text {tr }}$ for the 3 component. Hence a system, for which no fluorescence photons are detected, is described by the density matrix

$$
\rho_{\mathrm{d}}^{0}\left(\tau_{\mathrm{p}} ; \eta^{\prime}\right) \equiv P_{0}^{\mathrm{e}}\left(\tau_{\mathrm{p}} ;|\psi\rangle\right)|2\rangle\langle 2|+\left(P_{0}^{\mathrm{d}}\left(\tau_{\mathrm{p}} ;|\psi\rangle\right)-P_{0}^{\mathrm{e}}\left(\tau_{\mathrm{p}} ;|\psi\rangle\right)\right)|1\rangle\langle 1|
$$

where the normalization is such that the trace is $P_{0}^{\mathrm{d}}$, i.e. the probability of no detection. We call the $|1\rangle\langle 1|$ component the non-reduced part, and the smaller this is the better a reduction as in the projection postulate is realized. If one makes no photon measurements, i.e. $\eta^{\prime}=0$ and $P_{\mathrm{d}} \equiv 1$, then $\rho_{\mathrm{d}}^{0}\left(\tau_{\mathrm{p}} ; \eta^{\prime}\right)$ is just the diagonal density matrix of the Bloch equations which was discussed in Refs. [9, 10].

We will show further below (Section 4.1) that for $P_{0}^{\mathrm{d}}$ an equation analogous to Eq. (8) holds,

$$
P_{0}^{\mathrm{d}}(\tau ;|\psi\rangle)=|\langle 2 \mid \psi\rangle|^{2}+|\langle 1 \mid \psi\rangle|^{2} P_{0}^{\mathrm{d}}(\tau ;|1\rangle) .
$$

The density matrix in (18) then becomes, with Eq. (13) for $P_{0}^{\mathrm{e}}$,

$$
\rho_{\mathrm{d}}^{0}\left(\tau_{\mathrm{p}} ; \eta^{\prime}\right)=|\langle 2 \mid \psi\rangle|^{2}|2\rangle\left\langle\left. 2|+|\langle 1 \mid \psi\rangle\right|^{2} P_{0}^{\mathrm{d}}\left(\tau_{\mathrm{p}} ;|1\rangle\right) \mid 1\right\rangle\langle 1| \text {. }
$$


To determine the non-reduced part one therefore only has to calculate $P_{0}^{\mathrm{d}}\left(\tau_{\mathrm{p}} ;|1\rangle\right)$, the probability to detect no photon for initial state $|1\rangle$. This will be done in detail in Section 4 . Here we use a simplifying assumption, namely that for initial state $|1\rangle$ the fluorescence photons are emitted with a constant probability rate $I$ during the pulse,

$$
I=N_{\mathrm{e}}\left(\tau_{\mathrm{p}} ;|1\rangle\right) / \tau_{\mathrm{p}}
$$

For the detector the recorded rate is then

$$
I_{\eta^{\prime}}=\eta^{\prime} I=N_{\mathrm{d}}\left(\tau_{\mathrm{p}} ;|1\rangle\right) / \tau_{\mathrm{p}}
$$

Similar to radioactive decay Eq. (22) now implies

$$
\begin{aligned}
P_{0}^{\mathrm{d}}\left(\tau_{\mathrm{p}} ;|1\rangle\right) & =\mathrm{e}^{-I_{\eta^{\prime}} \tau_{\mathrm{p}}} \\
& =\mathrm{e}^{-N_{\mathrm{d}}\left(\tau_{\mathrm{p}} ;|1\rangle\right)} .
\end{aligned}
$$

Instead of a constant rate one can also assume the emission process to be Markovian. This assumption leads to the same result (cf., e.g., Ref. [24], p. 231).

We expect this to be a good approximation for small $\eta^{\prime}$ due to the following. For small $\eta^{\prime}$ most emitted photons go undetected, and to have a given average number $N_{\mathrm{d}}, N_{\mathrm{d}}=5$ say, one needs a large $N_{\mathrm{e}}$. The detected number of photons in this time interval has then an approximately Poissonian distribution, in accordance with Eq. (23). However, this argument gives no insight how small $\eta^{\prime}$ has to be or how large the error is. To answer this we need the more detailed analysis of Section 4.

From Eqs. (23) and (20) one obtains Eq. (11). Thus, with the above simplifying assumption of constant detection rate the non-reduced part decreases exponentially with $N_{\mathrm{d}}\left(\tau_{\mathrm{p}} ;|1\rangle\right)$. For a given detector one can adjust the laser parameters accordingly to come as close to the projection postulate as desired.

\section{General derivation}

\subsection{Solution by Laplace transforms}

With the results of Sections 2 and 3 the incorporation of detectors with overall efficiency $\eta^{\prime}<1$ is a purely statistical problem. After each emission the system (atom) is in its ground state, with all memory lost, and therefore the sequence of photon emissions is a renewal process [25], as is the sequence of photon detections. This renewal process (although derived from the Markovian quantum trajectories of state vectors) is not Markovian since it would then be a Poisson process [25, 26]. Hence the simplified treatment of Section 3 is only an approximation.

In view of Eq. (20) we have to determine $P_{0}^{\mathrm{d}}\left(\tau_{\mathrm{p}} ;|1\rangle\right)$ in order to estimate the quality of the reduction. For a system initially in $|\psi\rangle$ the probability to have exactly $N$ emissions occurring in $\left(\tau_{1}, \tau_{1}+\mathrm{d} \tau_{1}\right), \ldots,\left(\tau_{N}, \tau_{N}+\mathrm{d} \tau_{N}\right)$ in the interval $(0, \tau)$ is

$$
w_{1}^{\mathrm{e}}\left(\tau_{1} ;|\psi\rangle\right) \mathrm{d} \tau_{1} w_{1}^{\mathrm{e}}\left(\tau_{2}-\tau_{1} ;|1\rangle\right) \mathrm{d} \tau_{2} \cdots w_{1}^{\mathrm{e}}\left(\tau_{N}-\tau_{N-1} ;|1\rangle\right) \mathrm{d} \tau_{N} P_{0}^{\mathrm{e}}\left(\tau-\tau_{N} ;|1\rangle\right)
$$

and hence the probability to have exactly $N$ emissions in $(0, \tau)$, denoted by $P_{N}^{\mathrm{e}}(\tau ;|\psi\rangle)$, is for $N \geq 1$ obtained by integrating over the domain $0 \leq \tau_{1} \leq \tau_{2} \leq \ldots \leq \tau_{N} \leq \tau$. With Eq. (9) for $w_{1}^{\mathrm{e}}\left(\tau_{1} ;|\psi\rangle\right)$ this gives

$$
\begin{aligned}
P_{N}^{\mathrm{e}}(\tau ;|\psi\rangle)= & |\langle 1 \mid \psi\rangle|^{2} \int_{0}^{\tau} \mathrm{d} \tau_{N} P_{0}^{\mathrm{e}}\left(\tau-\tau_{N} ;|1\rangle\right) \int_{0}^{\tau_{N}} \mathrm{~d} \tau_{N-1} w_{1}^{\mathrm{e}}\left(\tau_{N}-\tau_{N-1} ;|1\rangle\right) \\
& \cdots \int_{0}^{\tau_{2}} \mathrm{~d} \tau_{1} w_{1}^{\mathrm{e}}\left(\tau_{2}-\tau_{1} ;|1\rangle\right) w_{1}^{\mathrm{e}}\left(\tau_{1} ;|1\rangle\right)
\end{aligned}
$$


For $N$ emitted photons the probability for all photons to go undetected is $\left(1-\eta^{\prime}\right)^{N}$, and therefore the probability of detecting no photon at all in $(0, \tau)$ for initial state $|\psi\rangle$ is

$$
P_{0}^{\mathrm{d}}(\tau ;|\psi\rangle)=P_{0}^{\mathrm{e}}(\tau ;|\psi\rangle)+\sum_{N=1}^{\infty}\left(1-\eta^{\prime}\right)^{N} P_{N}^{\mathrm{e}}(\tau ;|\psi\rangle) .
$$

Eq. (19) immediately follows from this when using Eqs. (25) and (13).

We now use Laplace transform, denoted by ${ }^{\wedge}$. The convolution theorem for Laplace transforms together with Eqs. (26) and (25) for $|\psi\rangle=|1\rangle$ gives

$$
\begin{aligned}
\hat{P}_{0}^{\mathrm{d}}(p ;|1\rangle) & =\hat{P}_{0}^{\mathrm{e}}(p ;|1\rangle) \sum_{N=0}^{\infty}\left(1-\eta^{\prime}\right)^{N} \hat{w}_{1}^{\mathrm{e}}(p ;|1\rangle) \\
& =\frac{\hat{P}_{0}^{\mathrm{e}}(p ;|1\rangle)}{1-\left(1-\eta^{\prime}\right) \hat{w}_{1}^{\mathrm{e}}(p ;|1\rangle)} .
\end{aligned}
$$

$\hat{P}_{0}^{\mathrm{e}}$ and $\hat{w}_{1}^{\mathrm{e}}$ are easily obtained from Eqs. (10) and (11) and yield $\hat{P}_{0}^{\mathrm{d}}$ as a quotient of the form

$$
\hat{P}_{0}^{\mathrm{d}}(p ;|1\rangle)=\frac{g(p)}{f_{\eta^{\prime}}(p)}
$$

where

$$
\begin{aligned}
f_{\eta^{\prime}}(p) & =p^{3}+\frac{3}{2} A p^{2}+\frac{1}{2}\left(A^{2}+2 \Omega^{2}\right) p+\frac{1}{2} \eta^{\prime} A \Omega^{2} \\
g(p) & =p^{2}+\frac{3}{2} A p+\frac{1}{2}\left(A^{2}+2 \Omega^{2}\right)=f_{0}(p) / p .
\end{aligned}
$$

The same expression was derived by somewhat different methods in Ref. [22] and the zeros of the denominator were found for the special case $\Omega=A / 2$.

Let $p_{1}, p_{2}, p_{3}$ be the zeros of $f_{\eta^{\prime}}(p)$. Either all of them are real and negative or one is negative and the other two are complex conjugates of each other, with negative real parts. We choose $p_{1}$ to be the real zero closest to zero and $\left|\operatorname{Re} p_{2}\right| \leq\left|\operatorname{Re} p_{3}\right|$. By partial fractions one then obtains, for distinct zeros,

$$
\begin{aligned}
P_{0}^{\mathrm{d}}(\tau ;|1\rangle) & =\sum_{i=1}^{3} \frac{g\left(p_{i}\right)}{\left(p_{i}-p_{i+1}\right)\left(p_{i}-p_{i+2}\right)} \mathrm{e}^{p_{i} \tau} \\
& \equiv c_{1} \mathrm{e}^{p_{1} \tau}+c_{2} \mathrm{e}^{p_{2} \tau}+c_{3} \mathrm{e}^{p_{3} \tau} .
\end{aligned}
$$

where we use $p_{i+3} \equiv p_{i}$. If two zeros coincide one has to take limits. An alternative form is obtained by noting that

$$
f_{\eta^{\prime}}(p) /\left(p-p_{i}\right)=\left(p-p_{i+1}\right)\left(p-p_{i+2}\right) .
$$

Letting $p \rightarrow p_{i}$, the 1.h.s. becomes $f_{\eta^{\prime}}^{\prime}\left(p_{i}\right)=f_{0}^{\prime}\left(p_{i}\right)$, and thus

$$
P_{0}^{\mathrm{d}}(\tau ;|1\rangle)=\sum_{i=1}^{3} \frac{g\left(p_{i}\right)}{f_{0}^{\prime}\left(p_{i}\right)} \mathrm{e}^{p_{i} \tau} .
$$

Furthermore, since

$$
f_{0}(p)=f_{\eta^{\prime}}(p)-\frac{1}{2} \eta^{\prime} A \Omega^{2}
$$

and $f_{\eta^{\prime}}\left(p_{i}\right)=0$, one has from Eq. (30)

$$
g\left(p_{i}\right)=-\frac{1}{2} \eta^{\prime} A \Omega^{2} / p_{i} .
$$


Again using $f_{\eta^{\prime}}\left(p_{i}\right)=0$ to recast $p_{i} f_{0}^{\prime}\left(p_{i}\right)$ one than finds

$$
c_{i}=\frac{g\left(p_{i}\right)}{f_{0}^{\prime}\left(p_{i}\right)}=\frac{\frac{1}{2} \eta^{\prime} A \Omega^{2}}{\frac{1}{2} \eta^{\prime} A \Omega^{2}-\frac{3}{2} A p_{i}^{2}-2 p_{i}^{3}} .
$$

We will next derive an approximate solution to Eq. (33) and will then discuss its range of validity.

\subsection{Approximate form of $P_{0}^{\mathrm{d}}$}

With $\mu_{1,2}$ as in Eq. (15) the zeros of $f_{\eta^{\prime}=0}(p)$ are

$$
\left(p_{1}, p_{2}, p_{3}\right)=\left(0,-\mu_{1},-\mu_{2}\right) \text {. }
$$

For small $\eta^{\prime}, p_{i}$ can be found perturbatively by linearization (Newton's method). In particular one finds

$$
p_{1} \cong-\eta^{\prime} \frac{A \Omega^{2}}{A^{2}+2 \Omega^{2}} .
$$

This can now be inserted into Eq. (36) for $c_{1}$, where in the denominator we neglect $\eta^{2}$ when compared to 1 and $\eta^{\prime}$. Eq. (16) for $N_{\mathrm{e}}\left(\tau_{\mathrm{p}} ;|1\rangle\right)$ is used to recast e ${ }^{p_{1} \tau_{\mathrm{p}}}$. With $N_{\mathrm{d}}=\eta^{\prime} N_{\mathrm{e}}$ we then obtain

$$
c_{1} \mathrm{e}^{p_{1} \tau_{\mathrm{p}}}=\frac{1}{1-3 \eta^{\prime} \frac{A^{2} \Omega^{2}}{\left(A^{2}+2 \Omega^{2}\right)^{2}}} \exp \left\{-N_{\mathrm{d}}-3 \eta^{\prime} \frac{A^{2} \Omega^{2}}{\left(A^{2}+2 \Omega^{2}\right)^{2}}\right\} .
$$

Expanding in $\eta^{\prime}$, the linear terms cancel and only $\eta^{\prime 2}$ terms with coefficients less than 1 remain; these are neglected when compared to 1.

From Eqs. (15) and (37) one sees that, for small $\eta^{\prime}, \mathrm{e}^{p_{2} \tau_{\mathrm{p}}}$ and $\mathrm{e}^{p_{3} \tau_{\mathrm{p}}}$ are much smaller in absolute value than $\mathrm{e}^{p_{1} \tau_{\mathrm{p}}}$. Therefore the second and third summands in Eq. (31) are neglected. (Some care is needed for $p_{2}$ close to $p_{3}$; this is discussed in detail in the next subsection.)

Thus we arrive at the approximate expression for small $\eta^{\prime}$,

$$
P_{0}^{\mathrm{d}}\left(\tau_{\mathrm{p}} ;|1\rangle\right)=\mathrm{e}^{-N_{\mathrm{d}}\left(\tau_{\mathrm{p}} ;|1\rangle\right)} .
$$

In view of the derivation $\eta^{\prime}$ has to be small enough for Newton's method to give a reliable $p_{1}$. In the next subsection the range of validity of the above expression will be discussed in detail. The reader not interested in all the details can proceed directly to the strict bound in Eq. (55).

\subsection{Validity range and precise estimates}

We now present upper and lower bounds on $P_{0}^{\mathrm{d}}$, and thus on the non-reduced part. These will justify the preceding approximate solution in more detail.

For this we will need more information on the zeros of the curve $y=f_{\eta^{\prime}}(p)$. This curve is obtained by moving the curve $y=f_{0}(p)$ upward by the amount $\frac{1}{2} \eta^{\prime} A \Omega^{2}$ or, alternatively, by moving the abscissa downward (see Fig. 2). We note further that, for $\eta^{\prime}=1$, the zeros are, in the given order,

$$
\left(p_{1}, p_{2}, p_{3}\right)= \begin{cases}\left(-2 \lambda_{1},-\frac{1}{2} A,-2 \lambda_{3}\right) & \text { for } \lambda_{1} \text { real } \\ \left(-\frac{1}{2} A,-2 \lambda_{1},-2 \lambda_{3}\right) & \text { otherwise }\end{cases}
$$

where $\lambda_{i}$ is given by Eq. (四). The point of inflexion of the curve is at $p_{\text {infl }}=-\frac{1}{2} A$, which is also a zero of $f_{1}(p)$, and this fixes the abscissa for $\eta^{\prime}=1$. Thus, from Fig. 2, $p_{1}$ lies between $p_{\text {infl }}$ and 0 . On the other hand, $p_{1}$ lies to the left of the tangent at $p=0$ (see Fig. 2), and so the r.h.s. of Eq. (38), i.e. Newton's method, provides an upper bound for $p_{1}$. One also verifies that

$$
-\eta^{\prime} \frac{A \Omega^{2}}{A^{2}+\Omega^{2}} \leq p_{1} \leq-\eta^{\prime} \frac{A \Omega^{2}}{A^{2}+2 \Omega^{2}} \quad \text { for } 0 \leq \eta^{\prime} \leq \frac{1}{3}
$$


by checking that $f_{\eta^{\prime}}(p)$ changes sign between these values.

As zeros of the polynomial $f_{\eta^{\prime}}$ the $p_{i}$ 's satisfy

$$
\begin{aligned}
p_{1}+p_{2}+p_{3} & =-\frac{3}{2} A \\
p_{1} p_{2}+p_{2} p_{3}+p_{1} p_{3} & =\frac{1}{2}\left(A^{2}+2 \Omega^{2}\right) .
\end{aligned}
$$

If $p_{2}$ and $p_{3}$ are real they must lie to the left of $p_{\text {infl }}$, i.e. $p_{2,3} \leq-\frac{1}{2} A$. If $p_{1}$ is the only real zero then $p_{3}=p_{2}^{*}$. Writing

$$
p_{2 / 3}=-a \mp \mathrm{i} b, \quad a, b>0
$$

one obtains from Eqs. (42) and (43)

$$
\begin{aligned}
a & =\frac{3}{4} A+\frac{1}{2} p_{1} \\
b^{2} & =\Omega^{2}-\frac{1}{16} A^{2}+\frac{3}{4} p_{1}^{2}+\frac{3}{4} A p_{1} .
\end{aligned}
$$

Now we discuss $c_{1} \mathrm{e}^{p_{1} \tau_{\mathrm{p}}}$, with $c_{1} \geq 0$ given by Eq. (36) for $i=1$. We replace $p_{1}$ in the exponent and $p_{1}^{3}$ in the denominator of $c_{1}$ by the r.h.s. of the inequality (41) and $p_{1}^{2}$ by the l.h.s. of (41). Then one obtains instead of Eq. (39), the inequality

$$
c_{1} \mathrm{e}^{p_{1} \tau_{\mathrm{p}}} \leq\left\{1-3 \eta^{\prime} \frac{A^{2} \Omega^{2}}{\left(A^{2}+\Omega^{2}\right)^{2}}+4 \eta^{\prime 2} \frac{A^{2} \Omega^{4}}{\left(A^{2}+2 \Omega^{2}\right)^{3}}\right\}^{-1} \exp \left\{-N_{0}^{\mathrm{d}}-3 \eta^{\prime} \frac{A^{2} \Omega^{2}}{\left(A^{2}+2 \Omega^{2}\right)^{2}}\right\}
$$

for $\eta^{\prime} \leq \frac{1}{3}$. Evaluating this graphically one finds, for arbitrary $A$ and $\Omega$ and for $\eta^{\prime} \leq 1 / 3$

$$
c_{1} \mathrm{e}^{p_{1} \tau_{\mathrm{p}}} \leq \frac{1}{1-.45 \eta^{\prime}} \exp \left\{-N_{0}^{\mathrm{d}}\right\}
$$

For $p_{2}$ approaching $p_{3}$ one sees from Eq. (31) that $c_{2}$ and $c_{3}$ diverge, but cancellations occur. To take these into account we use Eq. (35) to write

$$
c_{2} \mathrm{e}^{p_{2} \tau_{\mathrm{p}}}+c_{3} \mathrm{e}^{p_{3} \tau_{\mathrm{p}}}=-\frac{\frac{1}{2} \eta^{\prime} A \Omega^{2}}{p_{2} p_{3}\left(p_{2}-p_{1}\right)\left(p_{3}-p_{1}\right)} \frac{\left\{p_{3}\left(p_{3}-p_{1}\right) \mathrm{e}^{p_{2} \tau_{\mathrm{p}}}-p_{2}\left(p_{2}-p_{1}\right) \mathrm{e}^{p_{3} \tau_{\mathrm{p}}}\right\}}{p_{2}-p_{3}}
$$

and by recasting the expression in curly bracket as

$$
\left\{\left(p_{3}-p_{2}\right)\left(p_{3}+p_{2}-p_{1}\right) \mathrm{e}^{p_{2} \tau_{\mathrm{p}}}+\left(p_{2}^{2}-p_{2} p_{1}\right)\left(\mathrm{e}^{p_{2} \tau_{\mathrm{p}}}-\mathrm{e}^{p_{3} \tau_{\mathrm{p}}}\right)\right\}
$$

we obtain, with Eq. (42),

$$
c_{2} \mathrm{e}^{p_{2} \tau_{\mathrm{p}}}+c_{3} \mathrm{e}^{p_{3} \tau_{\mathrm{p}}}=-\frac{\frac{1}{2} \eta^{\prime} A \Omega^{2}}{p_{2} p_{3}\left(p_{2}-p_{1}\right)\left(p_{3}-p_{1}\right)}\left\{\left(\frac{3}{2} A+2 p_{1}\right) \mathrm{e}^{p_{2} \tau_{\mathrm{p}}}+\left(p_{2}^{2}-p_{2} p_{1}\right) \frac{\mathrm{e}^{p_{2} \tau_{\mathrm{p}}}-\mathrm{e}^{p_{3} \tau_{\mathrm{p}}}}{p_{2}-p_{3}}\right\}
$$

which remains manifestly finite for $p_{2} \rightarrow p_{3}$.

If all $p_{i}$ are real, then it is evident that the expression in the curly brackets as well as the fraction in front are positive so that the overall expression is negative. Hence its omission leads in this case to an upper bound for $P_{0}^{\mathrm{d}}$.

If $p_{2,3}$ are complex, $p_{2,3}=-a \mp \mathrm{i} b$, then the last equation can be written as

$$
\begin{aligned}
c_{2} \mathrm{e}^{p_{2} \tau_{\mathrm{p}}}+c_{3} \mathrm{e}^{p_{3} \tau_{\mathrm{p}}}= & -\frac{\frac{1}{2} \eta^{\prime} A \Omega^{2}}{\left(a^{2}+b^{2}\right)\left(a^{2}+b^{2}+2 a p_{1}+p_{1}^{2}\right)} \\
& \left\{\left(\frac{3}{2} A+2 p_{1}\right) \cos b \tau_{\mathrm{p}}-b \sin b \tau_{\mathrm{p}}+\left(a^{2}+a p_{1}\right) \frac{\sin b \tau_{\mathrm{p}}}{b}\right\} \mathrm{e}^{-a \tau_{\mathrm{p}}}
\end{aligned}
$$


From Eqs. (45) and (16) for $a$ and $N_{\mathrm{e}}$ one obtains

$$
\mathrm{e}^{-a \tau_{\mathrm{p}}} \leq \exp \left\{-N_{\mathrm{e}}-\left(\frac{3}{4} A+\frac{1}{2} p_{1}-\frac{A \Omega^{2}}{A^{2}+2 \Omega^{2}}\right) \tau_{\mathrm{p}}\right\} .
$$

In Eq. (52) we bound the absolute value of the expression in the curly brackets by using $\left|\sin b \tau_{\mathrm{p}} / b\right| \leq \tau_{\mathrm{p}}$ and $\left|\sin b \tau_{\mathrm{p}}\right|,\left|\cos b \tau_{\mathrm{p}}\right| \leq 1$. With Eq. (53) this leads to an upper bound of the form

$$
\left.\mathrm{e}^{-\gamma t}\right] \mathrm{e}^{-N_{\mathrm{e}}} \cdot \eta^{\prime} \text { const }\left[\left(\alpha+\beta \tau_{\mathrm{p}}\right) \mathrm{e}^{-\gamma \tau_{\mathrm{p}}}\right] \mathrm{e}^{-N_{\mathrm{e}}} .
$$

The maximum of the expression in the square brackets is taken for $\tau_{\mathrm{p}}=\tau_{\mathrm{m}}=\frac{1}{\gamma}-\frac{\alpha}{\beta}$ if $\tau_{\mathrm{m}} \geq 0$, otherwise for 0 . One can check graphically that $\tau_{\mathrm{m}}<0$ for $\eta^{\prime} \leq 1 / 3$ and hence the maximum is taken at $\tau_{\mathrm{p}}=0$. An upper bound, valid for all $A$ and $\Omega$, is then found graphically, with $N_{\mathrm{e}}=N_{\mathrm{d}} / \eta^{\prime}$ and for $\eta^{\prime} \leq 1 / 3$, as

$$
\left|c_{2} \mathrm{e}^{p_{2} \tau_{\mathrm{p}}}+c_{3} \mathrm{e}^{p_{3} \tau_{\mathrm{p}}}\right| \leq 0.81 \eta^{\prime} \mathrm{e}^{-N_{\mathrm{d}} / \eta^{\prime}}
$$

Together with the bound for $c_{1} \mathrm{e}^{p_{1} \tau_{\mathrm{p}}}$ in Eq. (48) this gives, for $\eta^{\prime} \leq 1 / 3$,

$$
P_{0}^{\mathrm{d}}\left(\tau_{\mathrm{p}} ;|1\rangle\right) \leq\left\{\frac{1}{1-0.45 \eta^{\prime}}+0.81 \eta^{\prime} \mathrm{e}^{-\left(\frac{1}{\eta^{\prime}}-1\right) N_{\mathrm{d}}}\right\} \mathrm{e}^{-N_{\mathrm{d}}} .
$$

The expression in the curly brackets can be evaluated graphically, and this leads, for $N_{\mathrm{d}} \geq 1$ and $\eta^{\prime} \leq 1 / 3$, to

$$
P_{0}^{\mathrm{d}}\left(\tau_{\mathrm{p}} ;|1\rangle\right) \leq\left(1+\frac{2}{3} \eta^{\prime}\right) \mathrm{e}^{-N_{\mathrm{d}}\left(\tau_{\mathrm{p}} ;|1\rangle\right)}
$$

In a similar way one can also obtain a lower bound for $P_{0}^{\mathrm{d}}$, but it is quicker to use Jensen's inequality [26] which gives

$$
\sum_{N=0}^{\infty}\left(1-\eta^{\prime}\right)^{N} p_{N} \geq\left(1-\eta^{\prime}\right)^{\bar{N}}
$$

With $\bar{N} \equiv N_{\mathrm{e}}$ Eq. (26) then yields

$$
\left(1-\eta^{\prime}\right)^{N_{\mathrm{e}}}=\mathrm{e}^{\ln \left(1-\eta^{\prime}\right) N_{\mathrm{e}}} \leq P_{0}^{\mathrm{d}} .
$$

Since $\left|\ln \left(1-\eta^{\prime}\right)\right| \leq \eta^{\prime}+\frac{1}{2} \eta^{\prime 2}$ one finally obtains, with $N_{\mathrm{d}} \equiv N_{\mathrm{d}}\left(\tau_{\mathrm{p}} ;|1\rangle\right)$ and $P_{0}^{\mathrm{d}} \equiv P_{0}^{\mathrm{d}}\left(\tau_{\mathrm{p}} ;|1\rangle\right)$,

$$
\mathrm{e}^{-\left(1+\eta^{\prime} / 2\right) N_{\mathrm{d}}} \leq\left(1-\eta^{\prime}\right)^{N_{\mathrm{d}} / \eta^{\prime}} \leq P_{0}^{\mathrm{d}} \leq\left(1+\frac{2}{3} \eta^{\prime}\right) \mathrm{e}^{-N_{\mathrm{d}}}
$$

where the 1.h.s. always holds and the r.h.s. for $N_{\mathrm{d}} \geq 1$ and $0 \leq \eta^{\prime} \leq 1 / 3$.

Eq. (57) not only shows that $P_{\mathrm{d}}^{0} \approx \mathrm{e}^{-N_{\mathrm{d}}}$ is indeed an excellent approximation for small detector efficiencies, as already suggested in Section 3, but also gives strict error bounds.

\section{Conclusions}

A short laser pulse driving the 1-3 transition brings about a reduction of a state of the 1-2 system in two ways as follows. If one or more fluorescence photons are emitted then the system ends up in $|1\rangle$, after a transient time for the decay of level 3. If no photons are emitted then the reduced (conditional) time development pushes the state towards $|2\rangle$. Its tiny $|1\rangle$ component can be estimated from above by

$$
|\langle 1 \mid \psi\rangle|^{2} \mathrm{e}^{-N_{\mathrm{e}}}
$$


Thus the reduction of $|\psi\rangle$ to $|2\rangle$ is excellent if the parameters of the pulse are such that $N_{\mathrm{e}} \geq 5$, i.e. if the average number of photons emitted by an atom initially in the ground state is 5 or more. If one measures with a detector which does not detect all photons $\left(\eta^{\prime}<1\right.$, e.g. solid angle less than $4 \pi$ ), then one has a problem for single systems.

Detecting no fluorescence photons during a pulse may be due either to no emission at all or to missing the emitted photons. As a consequence a system with initial state $|\psi\rangle$ for which no fluorescence photons are detected during a pulse is in a mixture of $|1\rangle$ and $|2\rangle$, namely

$$
|\langle 2 \mid \psi\rangle|^{2}|2\rangle\left\langle\left. 2|+|\langle 1 \mid \psi\rangle\right|^{2} P_{0}^{\mathrm{d}}\left(\tau_{\mathrm{p}} ;|1\rangle\right) \mid 1\right\rangle\langle 1| .
$$

$P_{0}^{\mathrm{d}}\left(\tau_{\mathrm{p}} ;|1\rangle\right)$ is the probability of detecting no fluorescence photons for a pulse, with the system initially in the ground state. We have expressed $P_{0}^{\mathrm{d}}$ in terms of the easily measurable quantity $N_{\mathrm{d}}\left(\tau_{\mathrm{p}} ;|1\rangle\right)$, the average number of detected photons per system, with initial state $|1\rangle . N_{\mathrm{d}}$ is simply determined from the fluorescence rate of an ensemble of systems initially in the ground state. For small efficiency $\eta^{\prime}$ we have shown that

$$
P_{0}^{\mathrm{d}}=\mathrm{e}^{-N_{\mathrm{d}}}
$$

and, more precisely, for $\eta^{\prime} \leq 1 / 3$ and $N_{\mathrm{d}} \geq 1$,

$$
\mathrm{e}^{-\left(1+\eta^{\prime} / 2\right) N_{\mathrm{d}}} \leq P_{0}^{\mathrm{d}} \leq\left(1+\frac{2}{3} \eta^{\prime}\right) \mathrm{e}^{-N_{\mathrm{d}}}
$$

Thus for increasing $N_{\mathrm{d}}$ the "non-reduced part" becomes very small, e.g. for $N_{\mathrm{d}}=3$ it is already less than $5 \%$.

Depending on the desired quality of the reduction one can adjust the parameters of the laser pulse in a simple way to give the required value of $N_{\mathrm{d}}$.

If one considers not a single system but an ensemble of atoms the detector plays no role. After the pulse the state is reduced to a mixture of $|1\rangle$ and $|2\rangle$ regardless of whether all fluorescence photons are actually observed or not. This can also be seen by the use of Bloch equations [9, 10].

If the 1-2 transition is driven by an additional interaction during the short laser pulse then complications arise and modifications to the projection-postulate result as obtained above have to be incorporated [6, 7].

Acknowledgments. We would like to thank D.G. Sondermann for stimulating discussions. 


\section{References}

[1] Lüders, G., 1951, Ann. d. Phys., 8, 323

[2] von Neumann, J., 1932, Mathematische Grundlagen der Quantenmechanik (Berlin: Springer), English translation: 1955, Mathematical Foundations of Quantum Mechanics (Princeton: University Press), Chapter V.1.

[3] Dirac, P. A. M., 1930, The Principles of Quantum Mechanics (Oxford: Clarendon Press)

[4] Cook, R. J., 1988, Phys. Scr., T 21, 49

[5] Itano, W. M., Heinzen, D. J., Bollinger, J. J., and Wineland, D. J., 1990, Phys. Rev. A, 41, 2295

[6] Beige, A. and Hegerfeldt, G. C., 1996, Phys. Rev. A, 53, 53; quant-ph/9512012

[7] Beige, A., Hegerfeldt, G. C., and Sondermann, D. G., 1996, Quantum Semiclass. Opt. (in press); quant-ph/9607006

[8] Petrosky, T., Tasaki, S., and Prigogine, I., 1990, Phys. Lett. A, 151, 109

Petrosky, T., Tasaki, S., and Prigogine, I., 1991, Physica A, 170, 306

[9] Block, E. and Berman, P. R., 1991, Phys. Rev. A, 44, 1466

[10] Frerichs, V. and Schenzle, A., 1991, Phys. Rev. A, 44, 1962

[11] Gagen, M. J. and Milburn, G. J., 1993, Phys. Rev. A, 47, 1467

[12] Power, W. L. and Knight, P. L., 1996, Phys. Rev. A, 53, 1052

[13] Mahler, G., and Weberruß, V. A., 1995, Quantum Networks: Dynamics of Open Nanostructures (Berlin: Springer)

Keller, M., and Mahler, G., 1996, Quantum Semiclass. Opt., 8, 223

[14] Hegerfeldt, G. C. and Wilser, T. S., 1992 in: Classical and Quantum Systems. Proceedings of the II. International Wigner Symposium, Goslar July 1991 (ed. by H.D. Doebner, W. Scherer, and F. Schroeck) (Singapur: World Scientific), p. 104

[15] Wilser, T. S., 1991, Doctoral Dissertation, University of Göttingen (Göttingen)

[16] Hegerfeldt, G. C., 1993, Phys. Rev. A, 47, 449

[17] Hegerfeldt, G. C. and Sondermann, D. G., 1996, Quantum Semiclass. Opt., 8, 121; atom-ph/9512003

[18] Carmichael, H., 1993, An Open Systems Approach to Quantum Optics, Lecture Notes in Physics (Berlin: Springer)

[19] Reibold, R., 1993, J. Phys. A, 26, 179

[20] Plenio, M. B., 1994, Doctoral Dissertation, University of Göttingen (Göttingen) 
[21] Hegerfeldt, G. C., and Plenio, M. B., 1996, Phys. Rev. A, 53, 1164

[22] Carmichael, H. J., Singh, S., Vyas, R., and Rice, P. R., 1989, Phys. Rev. A, 39, 1200

[23] Dalibard, J., Castin, Y., and Mølmer, K., 1992, Phys. Lett., 68, 580

[24] Loudon, R., 1988, The Quantum Theory of Light (Oxford: Clarendon Press)

[25] Cox, D. R., 1962, Renewal Theory (London: Methuen)

[26] Breiman, L., 1968, Probability (Reading, Massachusetts: Addison-Wesley Publishing Co) 


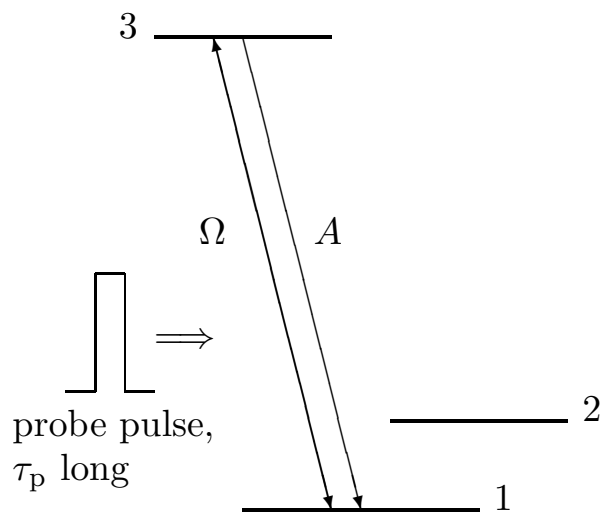

Figure 1: V system with level 2 and auxiliary level 3 with Einstein coefficient $A . \Omega$ is the Rabi frequencies of the probe pulse of length $\tau_{\mathrm{p}}$.

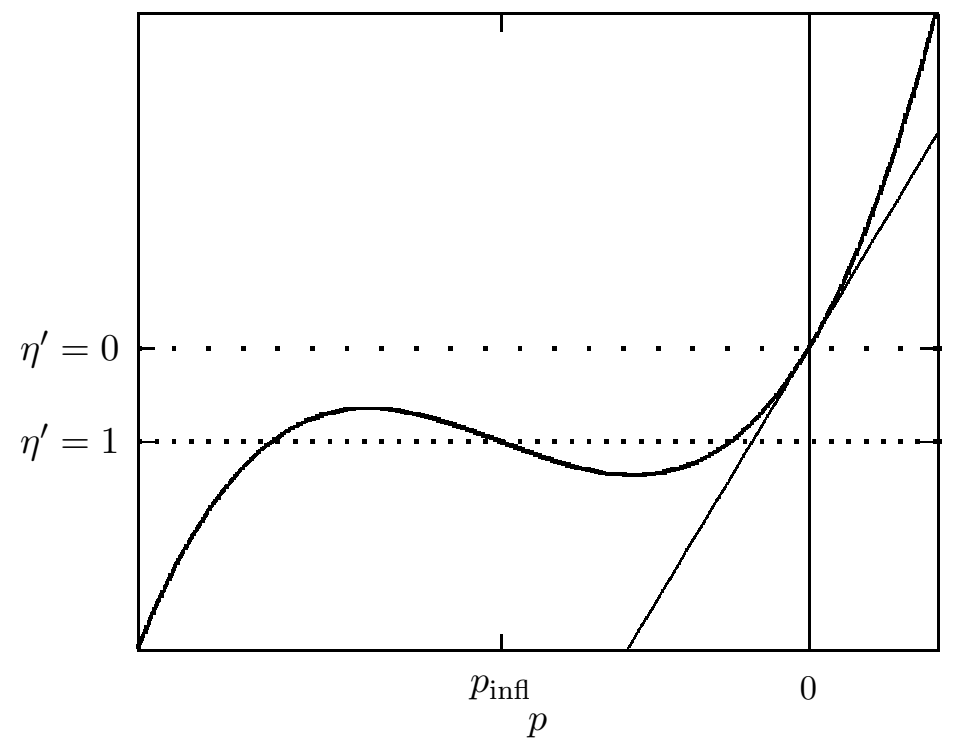

Figure 2: Typical form of $f_{\eta^{\prime}}(p)$. With increasing $\eta^{\prime}$ the abcissa shifts downwards and passes for $\eta^{\prime}=1$ through the point of inflection at $p_{\text {infl. }}$. 